\title{
Observation of optical absorption correlated with surface state of topological insulator
}

\author{
Jiwon Jeon, ${ }^{1}$ Kwangnam Yu, ${ }^{1}$ Jiho Kim,,${ }^{1}$ Jisoo Moon, ${ }^{2}$ Seongshik Oh, ${ }^{2}$ and E. J. Choi ${ }^{1, *}$ \\ ${ }^{1}$ Department of Physics, University of Seoul, Seoul 130-743, Korea \\ ${ }^{2}$ Department of Physics and Astronomy, Rutgers, \\ the State University of New Jersey, Piscataway, New Jersey 08854, USA
}

(Dated: June 6, 2022)

\begin{abstract}
We performed broadband optical transmission measurements of $\mathrm{Bi}_{2} \mathrm{Se}_{3}$ and In-doped $\left(\mathrm{Bi}_{1-\mathrm{x}} \operatorname{In}_{\mathrm{x}}\right)_{2} \mathrm{Se}_{3}$ thin films, where in the latter the spin-orbit coupling (SOC) strength can be tuned by introducing In. Drude and interband transitions exhibit In-dependent changes that are consistent with evolution from metallic $(\mathrm{x}=0)$ to insulating $(\mathrm{x}=1)$ nature of the end compounds. Most notably, an optical absorption peak located at $\hbar \omega=1 \mathrm{eV}$ in $\mathrm{Bi}_{2} \mathrm{Se}_{3}$ is completely quenched at $\mathrm{x}=0.06$, the critical concentration where the phase transition from TI into non-TI takes place. For this $\mathrm{x}$, the surface state (SS) is vanished from the band structure as well. The correlation between the $1 \mathrm{eV}$ optical peak and the SS in the x-dependences suggests that the peak is associated with the SS. We further show that when $\mathrm{Bi}_{2} \mathrm{Se}_{3}$ is electrically gated, the $1 \mathrm{eV}$-peak becomes stronger(weaker) when electron is depleted from (accumulated into) the SS. These observations combined together demonstrate that under the $\hbar \omega=1 \mathrm{eV}$ illumination electron is excited from a bulk band into the topological surface band of $\mathrm{Bi}_{2} \mathrm{Se}_{3}$. The optical population of surface band is of significant importance not only for fundamental study but also for TI-based optoelectronic device application.
\end{abstract}

Introduction. Topological insulator (TI) is a novel state of matter characterized by insulating bulk and metallic surface 1 15 . The surface state, topologically protected and chirally-textured, supports dissipationless spin-conserving current, applicable for quantum devices 6 8]. Optically, various kinds of electron transitions occur in a TI under photo illumination both in the surface and in the bulk as demonstrated by numerous previous experiments: For the archetypal TI material $\mathrm{Bi}_{2} \mathrm{Se}_{3}$, intraband (Drude) transition and Kerr rotation of the surface carrier were observed in $\mathrm{THz}$ measurement [9]. In the infrared range, Post et al measured the interband transition from bulk valence-band (VB) to bulk conduction-band $(\mathrm{CB}), \mathrm{VB} \rightarrow \mathrm{CB}$, and determined upper bound of Drude weight of SS [10. Also Falsetti et al observed the infrared Berreman resonance of the surface electron in $\mathrm{Bi}_{2} \mathrm{Se}_{3}$ thin films [1]. On the other hand, for periodically modulated $\mathrm{Bi}_{2} \mathrm{Se}_{3}$, plasmonic excitation of the surface electron 12 and the plasmon-phonon interaction 13 were observed at $\mathrm{THz}$ frequencies.

One interesting optical absorption that TI can host yet has not been detected is an excitation of electron from bulk band into SS. This particular transition between bulk and surface, $\mathrm{VB} \rightarrow \mathrm{SS}$, will provide rare opportunity to study how the surface and bulk are connected optically. Also, when the surface electron is populated by this optical transition, the surface electron is increased and therefore the topological current ( $\propto$ surface electron) is enhanced, which can boost the performance of TI as optoelectronic device such as photogalvanics and optical imaging display [14, 15. In fact Sobota et al showed that a similar transition can occur from bulk CB to 2nd SS, $\mathrm{CB} \rightarrow 2 \mathrm{nd} \mathrm{SS}$, at a visible frequency [16]. (Here the 2 nd SS

\footnotetext{
* echoi@uos.ac.kr
}

refers to another SS that lies above the 1st or fundamental SS). However the optical transition into the 1st SS or fundamental $\mathrm{SS}, \mathrm{VB} \rightarrow \mathrm{SS}$, was not reported yet. It is not clear at this point whether the lack of the SS-populating optical transition is due to that other transition such as $\mathrm{VB} \rightarrow \mathrm{CB}$ is overwhelmingly stronger, making the detection difficult [17, or more fundamentally, this particular transition is forbidden by optical selection rule.

Here we performed broadband optical absorption measurement of $\mathrm{Bi}_{2} \mathrm{Se}_{3}$ and $\left(\mathrm{Bi}_{1-\mathrm{x}} \mathrm{In}_{\mathrm{x}}\right)_{2} \mathrm{Se}_{3}$ thin films from far-IR to UV frequencies. In $\left(\mathrm{Bi}_{1-\mathrm{x}} \mathrm{In}_{\mathrm{x}}\right)_{2} \mathrm{Se}_{3}$, the spin-orbit coupling (SOC) strength is modulated by means of the indium (In) substitution. As In-content is increased the SOC of $\mathrm{Bi}_{2} \mathrm{Se}_{3}$ is decreased and consequently the topological property is softened. Eventually, at certain substitution level, the topological SS is completely quenched from the band structure. Accordingly any SS-related optical transition will be removed from the wide-range optical excitation spectrum, which in fact offers us an invaluable means to find the SS-population transition in particular. Our measurement shows signatures that such optical absorption may exist.

Experiment. High quality epitaxial $\mathrm{Bi}_{2} \mathrm{Se}_{3}$ and $\left(\mathrm{Bi}_{1-\mathrm{x}} \mathrm{In}_{\mathrm{x}}\right)_{2} \mathrm{Se}_{3}$ thin films were grown on $\mathrm{Al}_{2} \mathrm{O}_{3}$ and $\mathrm{SiO}_{2} / \mathrm{Si}$ substrates using the MBE method [18. Optical transmittance $\mathrm{T}(\omega)$ was measured from Far-infrared to UV by using Fourier transform infrared spectroscopy (FTIR) spectrometer in combination with spectroscopic ellipsometer. For gate-dependent optical measurement gate-voltage $V_{\mathrm{G}}$ was applied between $\mathrm{Bi}_{2} \mathrm{Se}_{3}$ and $\mathrm{Si}$ of the substrate. Optical conductivity $\sigma_{1}(\omega)$ was calculated from transmission data through rigorous Kramers-Kronig transformation by using RefFit [19. The experimental details are described in Supplemental Material 1[20] and references therein. 
Results. Figure 1 shows the wide-range optical conductivity $\sigma_{1}(\omega)$ of the $50 \mathrm{QL}$-thick $\mathrm{Bi}_{2} \mathrm{Se}_{3}$ film. In the Far-infrared region, $\sigma_{1}(\omega)$ consists of Drude absorption and optical phonon peak, both coming from the bulk, where the former one arises from the Se-vacancy driven carrier [21, 22]. For $\hbar \omega>0.25 \mathrm{eV}$, the interband (IB) transition $\mathrm{VB} \rightarrow \mathrm{CB}$ leads to the rapid rise of $\sigma_{1}(\omega)$. Note that there is an absorption peak at $\hbar \omega=1 \mathrm{eV}$ ( $\equiv$ Peak-A hereafter) which we will pay particular attention to.

In Figure 2 we show optical conductivity measured for a series of In-substituted $\left(\mathrm{Bi}_{1-\mathrm{x}} \mathrm{In}_{\mathrm{x}}\right)_{2} \mathrm{Se}_{3}$ films. The In-concentration $x$ was varied for $0 \leq \mathrm{x} \leq 0.9$ range. Previous studies showed that as $\mathrm{Bi}$ is replaced by the light element In, the spin-orbit interaction is reduced and the topological property of $\mathrm{Bi}_{2} \mathrm{Se}_{3}$ becomes weaker 23 26]. At a critical concentration $\mathrm{x}_{c}$, phase transition from TI to non-TI (NTI) phase occurs where the bulk band gap is closed, and $\mathrm{CB}$ and VB begin to reinvert. The $\mathrm{x}_{c}$ lies between $\mathrm{x}=0.04$ and $\mathrm{x}=0.06$ depending on the film thickness, and for at $\mathrm{x} \geq \mathrm{x}_{c}$ the topological SS is completely vanished 223, 24]. The $\sigma_{1}(\omega)$ shows that Peak-A becomes weaker as $x$ increases. For quantitative analysis of this behavior we isolate Peak-A by removing background conductivity from $\sigma_{1}(\omega)$ as $\sigma_{1}^{A}(\omega)=\sigma_{1}(\omega)-\sigma_{1}^{B G}(\omega)$ as illustrated in the inset of Figure 2d, (a polynomial function was used for the $\sigma_{1}^{B G}$ ) and calculated the strength of Peak-A as $S=\int \sigma_{1}^{A}(\omega) d \omega$. Figure $2 \mathrm{~d}$ shows that $S$ is quenched at $\mathrm{x}=0.06$. To double check this behavior we performed independent analysis of Peak-A: we calculate the second derivative $\frac{d^{2} \sigma_{1}}{d E^{2}}$ and measure the distance $(\mathrm{w})$ and depth $(d)$ of the extrema pattern, which allows determination of strength $S\left(=\frac{1}{12} \sqrt{\frac{\pi}{6}} \cdot \mathrm{dw}^{3}\right)$ as well as width $\left(=\frac{1}{\sqrt{3}} \cdot \mathrm{w}\right)$ and height $\left(=\frac{1}{12} \cdot \mathrm{dw}^{2}\right)$ of Peak-A. (See Supplemental Fig. $\mathrm{S} 1$ for details 20]). The $S$ is quenched at $\mathrm{x}=0.06$ again, which confirms the $S=\int \sigma_{1}^{A}(\omega) d \omega$ analysis. Importantly $\mathrm{x}=0.06$ is the critical $\mathrm{x}_{c}$ for the $\mathrm{TI} \rightarrow \mathrm{NTI}$ transition for the thickness $d=50 \mathrm{QL}$ of our films: that is, the $\mathrm{SS}$ is vanished at this $\mathrm{x}$. This correlation of Peak-A with the TI $\rightarrow$ NTI transition strongly suggests that Peak-A is related with the topological SS of $\mathrm{Bi}_{2} \mathrm{Se}_{3}$. We emphasize that this behavior is strikingly different from those of the other optical absorption features: In Supplemental Fig.S2 20, we show that the Drude absorption is vanished at $\mathrm{x} \sim 0.5$, the phonon peak splits at $\mathrm{x}=0.12$, and the IB survives up to $\mathrm{x}=0.9$. (For $\mathrm{x}=1$, $\mathrm{In}_{2} \mathrm{Se}_{3}$ is a large gap band insulator with $E_{\mathrm{g}}>1.5 \mathrm{eV}$ ) Note that none of these features are correlated with $\mathrm{x}_{c}$. In contrast Peak-A manifests clear correlation with $\mathrm{x}_{c}$ and is the only absorption of such kind.

Given the correlation of Peak-A with SS, one can propose possible pictures on how Peak-A is created. Specifically, Peak-A can arise when (1) SS electron is excited into empty state lying $1 \mathrm{eV}$ above, or alternatively (2) electron lying at $1 \mathrm{eV}$ below is excited into the SS. In both scenarios Peak-A becomes extinct when
SS is suppressed at $x_{c}$. To find out which scenario is correct, we performed electrical gating experiment on the $\mathrm{Bi}_{2} \mathrm{Se}_{3}$ film $(d=8 \mathrm{QL})$. For this a $\mathrm{Bi}_{2} \mathrm{Se}_{3}$ film was grown on $\mathrm{SiO}_{2} / \mathrm{Si}$ substrate and optical transmission was measured while gate voltage $V_{\mathrm{G}}$ is applied between the film and $\mathrm{Si}$. In this back-gate configuration, the Fermi energy $\mathrm{E}_{F}$ shifts down (up) for the negative (positive) $V_{\mathrm{G}}$ due to electron depletion (accumulation) in the film. Fig.3(b) shows that $\mathrm{T}\left(V_{\mathrm{G}}\right) / \mathrm{T}(0)$ changes in the Far-IR, mid-IR, and at $1 \mathrm{eV}$. Figure 3(c) shows that Peak-A becomes stronger (weaker) for negative (positive) $V_{\mathrm{G}}$. Such change supports the scenario (2) over (1) for the following reason: For $V_{\mathrm{G}}<0$ the electron occupation of SS is reduced and more empty SS become available, which strengthens the transition of (2), which agrees with the increase of Peak-A strength. This relation is visualized in Fig.4. In the scenario (1), on the other hand, the surface electron is decreased and the peak becomes weaker, opposite to the observed behavior of Peak-A. Therefore, the $V_{\mathrm{G}}$-dependent result demonstrates that Peak-A arises most likely by excitation of electron from a state lying $1 \mathrm{eV}$ below into the SS. In this transition electron occupation of SS is increased, or equally, the SS is optically populated by illuminating $\mathrm{Bi}_{2} \mathrm{Se}_{3}$ with $\hbar \omega=1 \mathrm{eV}$. Here we remark that the $V_{\mathrm{G}}$-dependent change in Figure $3 \mathrm{c}$ is very small, less than even $0.1 \%$. Nevertheless the Peak-A change is successfully measured, demonstrating the superior sensitivity and stability of our experiment. For later analysis we calculate the $V_{\mathrm{G}}$-dependent $\sigma_{1}(\omega)$ from the $\mathrm{T}\left(V_{\mathrm{G}}\right) / \mathrm{T}(0)$ data $\left.27-29\right]$ and show it in Figure 3(d).

With the nature of Peak-A been identified, the next question to be addresed is the origin of the initial state. Before we discuss this issue, we give further thoughts on the gate-dependent growth of Peak-A. Figure 3 implies that the SS-population will become stronger if $\mathrm{E}_{F}$ could be brought down further. The latter would be possible when $V_{\mathrm{G}}$ is applied to high value beyond the limit of our measurement, where such high-gating was in fact demonstrated experimentally 30. Here we will consider how large Peak-A will grow in the strong-gating regime. In Figure $4 \mathrm{a}$, we show the bulk interband transition in the mid-IR range. The onset energy $\left(\equiv \mathrm{E}_{o p}\right)$ of this transition corresponds to the thick arrow in Fig.4(b). The $\mathrm{E}_{o p}$ increases when the Fermi level shifts up. Fig.4(c) shows that the increase rate is $\mathrm{dE}_{o p} / \mathrm{d} V_{\mathrm{G}}=1.74 \times 10^{-4}[\mathrm{eV} / \mathrm{V}] \equiv a$. In the mean time the $S=\int \sigma_{1}^{A}(\omega) d \omega$ calculated from Figure3(d) decreases at the rate $\mathrm{d} s / \mathrm{d} V_{\mathrm{G}}=-6.06 \times 10^{-4}[1 / \mathrm{V}] \equiv b$, where $s=\mathrm{S} / \mathrm{S}(0 \mathrm{~V})$ is the normalized $S$ by ungated $S(0)$. Given $a$ and $b$ we can eliminate $V_{\mathrm{G}}$ and obtain the $\mathrm{S}$ change against $\mathrm{E}_{F}$ as $\mathrm{d} s / \mathrm{dE}_{F}=\left(\mathrm{d} s / \mathrm{d} V_{\mathrm{G}}\right) \cdot\left(\mathrm{d}_{\mathrm{G}_{\mathrm{G}}} / \mathrm{dE}_{F}\right)=$ $b /(a / 2)=6.96[1 / \mathrm{eV}]$. Here $\mathrm{d} V_{\mathrm{G}} / \mathrm{dE}_{F}=(a / 2)^{-1}$ was derived by utilizing the Bernstein-Moss relation 31 33, namely, $\mathrm{dE}_{F} / \mathrm{d} V_{G}=\frac{1}{2} \times \mathrm{dE}_{o p} / \mathrm{d} V_{\mathrm{G}}=\frac{a}{2}$ where the factor $\frac{1}{2}$ comes from $\frac{1}{m_{C V}^{*}}=\frac{1}{m_{C B}^{*}}+\frac{1}{m_{V B}^{*}}$ and $m_{C B}^{*}=m_{V B}^{*}$ [34]. This result $\mathrm{d} s / \mathrm{dE}_{F}=6.96[1 / \mathrm{eV}]$ enables us estimate 
$S$ at high gating: For the pristine, electron-doped $\mathrm{Bi}_{2} \mathrm{Se}_{3}$ films like ours, the Fermi level lies typically at $E_{F} \sim 0.1 \mathrm{eV}$ from the $\mathrm{CB}$ bottom (CBB). If the gating shifts $E_{F}$ down to the $\mathrm{CBB}$, i.e, $\Delta \mathrm{E}_{F}=0.1$, then $s$ will increase approximately by $\Delta s \approx\left[\frac{d s}{d \mathrm{E}_{\mathrm{F}}}\right] \cdot \Delta \mathrm{E}_{F}=0.69$. that is, Peak-A grows by $\sim 70 \%$ compared with the ungated strength. If $\mathrm{E}_{F}$ is shifted further to the Dirac point, the latter lying $\sim 0.2 \mathrm{eV}$ from the $\mathrm{CBB}$, we have $s=s(0)+\Delta s \approx 1+\left[\frac{d s}{d \mathrm{E}_{\mathrm{F}}}\right] \cdot(0.1+0.2)=3.09$. That is, Peak-A grows as large as three-times. (Here we assumed $\frac{d s}{d \mathrm{E}_{\mathrm{F}}}$ is constant for simplicity, neglecting its $E_{F}$-dependence.) This estimation shows that substantial increase of the peak will occur at high-gating. From this exercise we also learn that if $S$ is precisely characterized as function of $\mathrm{E}_{F}$, it could be used to determine the location of the Fermi level in $\mathrm{Bi}_{2} \mathrm{Se}_{3}$ films, whereas usually more difficult ARPES should be performed.

Discussion. We now search for possible candidate for the initial state of the Peak-A. For this we refer to the band structure of $\mathrm{Bi}_{2} \mathrm{Se}_{3}$ reported in experimental [3, 7, 35]39] and theoretical [40 43] literatures, and schematically redrew it in Figure5. In Figure 5 note that there is an energy branch lying $\sim 1 \mathrm{eV}$ below the SS. Interestingly, this branch $\mathrm{E}(\mathrm{k})$ runs in near-parallel with the SS. If we consider the optical transition from this $\mathrm{E}(\mathrm{k})$ branch $(=\mathrm{i})$ to $\mathrm{SS}(=\mathrm{f})$, their parallel dispersion $\nabla_{k} \mathrm{E}^{\mathrm{i}}(\mathrm{k}) \cong \nabla_{k} \mathrm{E}^{\mathrm{f}}(\mathrm{k})$ leads to strong absorption due to that transition strength $\mathrm{S} \sim \int \frac{M_{f i}}{\left|\nabla_{k} \mathrm{E}^{\mathrm{f}}(\mathrm{k})-\nabla_{k} \mathrm{E}^{\mathrm{i}}\right|} d^{2} k$ becomes divergent. This yields a pronounced absorption at $\hbar \omega=\mathrm{E}_{f^{-}}$ $\mathrm{E}_{i}=1 \mathrm{eV}$, which agrees with the profile of Peak-A. (Here the transition matrix element $\mathrm{M}_{f i}$ is assumed to be constant for simplicity.) Therefore this $1 \mathrm{eV}-\mathrm{E}(\mathrm{k})$ is a plausible candidate for the i-state. We think that this assignment can be confirmed when theoretical calculation of $\sigma_{1}(\omega)$, not available currently, is performed. We remark that optical transition of $\mathrm{Bi}_{2} \mathrm{Se}_{3}$ between bulk and surface in particular was poorly studied so far with a rare exception [17. To consider another candidate native defect such as Se-vacancy can produce defect levels below $E_{F}$. However their energy locations are not well known, and generally such localized, dispersionless levels do not fulfill the $\nabla_{k} \mathrm{E}^{\mathrm{i}}(\mathrm{k}) \cong \nabla_{k} \mathrm{E}^{\mathrm{f}}(\mathrm{k})$ condition. We emphasize that, while supporting works should follow to definitely identify the $1 \mathrm{eV}$-bulk $\mathrm{E}(\mathrm{k})$ as origin of i-state, the occurrence of the optical population of $\mathrm{SS}$ in $\mathrm{Bi}_{2} \mathrm{Se}_{3}$ is evident judging from the properties of Peak-A we have unveiled regardless of the i-state origin.
To make further remark on the $\mathrm{T}\left(V_{\mathrm{G}}\right) / \mathrm{T}(0)$ data, Fig.3(b) shows that gate-dependent change occurs in the Far-IR and mid-IR ranges also. Similar change was reported for bulk-insulating $\left(\mathrm{Bi}_{1-\mathrm{x}} \mathrm{Sb}_{\mathrm{x}}\right)_{2} \mathrm{Te}_{3}$ films 44 . While for $\left(\mathrm{Bi}_{1-\mathrm{x}} \mathrm{Sb}_{\mathrm{x}}\right)_{2} \mathrm{Te}_{3}$ the mid-IR modulation peaks at $\sim 0.3 \mathrm{eV}$, the modulation for $\mathrm{Bi}_{2} \mathrm{Se}_{3}$ occurs at higher energy, peaked at $0.45 \mathrm{eV}$. This difference is attributed to that the interband transition taking place at $\mathrm{E}_{o p}=\mathrm{E}_{g}+2 \mathrm{E}_{F}$ is higher for $\mathrm{Bi}_{2} \mathrm{Se}_{3}$ where $\mathrm{E}_{F}$ is significant $(\sim 0.1 \mathrm{eV})$ compared with the insulating $\left(\mathrm{Bi}_{1-\mathrm{x}} \mathrm{Sb}_{\mathrm{x}}\right)_{2} \mathrm{Te}_{3}$ where $\mathrm{E}_{F}$ is considered to be much lower. Also, while the modulations in mid-IR and far-IR inevitably contain contribution from both bulk and surface states, the modulation strength of the $1 \mathrm{eV}$ feature is weaker, which may further support the surface-related origin. Further quantitative analysis and comparison will be published separately.

In conclusion we performed broadband optical absorption measurement on pristine $\mathrm{Bi}_{2} \mathrm{Se}_{3}$ and In-substituted $\left(\mathrm{Bi}_{1-\mathrm{x}} \mathrm{In}_{\mathrm{x}}\right)_{2} \mathrm{Se}_{3}$ thin films, as well as electrically gated $\mathrm{Bi}_{2} \mathrm{Se}_{3}$. The absorption Peak-A that occurs at $\hbar \omega=1 \mathrm{eV}$ showed clear correlation with the In-driven TI-NTI phase transition: it is activated at $\mathrm{x}<\mathrm{x}_{c}$ (TI-phase) but is completely vanished for $\mathrm{x}>\mathrm{x}_{c}$ (NTI) along with the quenching of the topological surface state. Furthermore, the Peak-A become stronger/weaker by the electron depletion/injection into the $\mathrm{Bi}_{2} \mathrm{Se}_{3}$ in the electrical gating measurement. The two experimental results provide convincing evidence that Peak-A arises from the population of SS, i.e, the optical excitation of electron from $1 \mathrm{eV}$ below into into SS. This SS-optical population increases the density of the surface electron, thus can enhance the topological electrical conduction, which promotes TI device application. Similar optically driven SS-population may be realized in other TI materials as well, which should be investigated in the future. Note added: For our $\left(\mathrm{Bi}_{1-\mathrm{x}} \mathrm{In}_{\mathrm{x}}\right)_{2} \mathrm{Se}_{3}$ films, the bulk transition $\mathrm{E}_{o p}$ shows a different $\mathrm{x}$-dependent behavior from Ref. 25]. It may come from that carrier doping due to Se-vacancy is sample-dependent for these TI films. See Supplemental Fig.S3[20].

Acknowledgments. This work was supported by the 2016 sabbatical year research grant of the University of Seoul. JM and SO are supported by the Gordon and Betty Moore Foundations EPiQS Initiative (GBMF4418) and National Science Foundation (NSF) grant EFMA1542798 .
[1] L. Fu, C. L. Kane, and E. J. Mele, Physical Review Letters 98, 106803 (2007).

[2] Y. Xia, D. Qian, D. Hsieh, L. Wray, A. Pal, H. Lin, A. Bansil, D. Grauer, Y. S. Hor, R. J. Cava, et al., Nature Physics 5, 398 (2009).

[3] M. Z. Hasan and C. L. Kane, Reviews of Modern Physics
82, 3045 (2010).

[4] J. E. Moore, Nature 464, 194 (2010).

[5] X.-L. Qi and S.-C. Zhang, Reviews of Modern Physics 83, 1057 (2011).

[6] P. Roushan, J. Seo, C. V. Parker, Y. S. Hor, D. Hsieh, D. Qian, A. Richardella, M. Z. Hasan, R. J. Cava, and 
A. Yazdani, Nature 460, 1106 (2009).

[7] D. Hsieh, Y. Xia, D. Qian, L. Wray, J. Dil, F. Meier, J. Osterwalder, L. Patthey, J. Checkelsky, N. Ong, et al., Nature 460, 1101 (2009).

[8] C. Li, O. Vant Erve, J. Robinson, Y. Liu, L. Li, and B. Jonker, Nature Nanotechnology 9, 218 (2014).

[9] R. V. Aguilar, A. Stier, W. Liu, L. Bilbro, D. George, N. Bansal, L. Wu, J. Cerne, A. Markelz, S. Oh, et al., Physical Review Letters 108, 087403 (2012).

[10] K. Post, B. Chapler, M. Liu, J. Wu, H. Stinson, M. Goldflam, A. Richardella, J. Lee, A. Reijnders, K. Burch, et al., Physical Review Letters 115, 116804 (2015).

[11] E. Falsetti, A. Nucara, P. P. Shibayev, M. Salehi, J. Moon, S. Oh, J.-B. Brubach, P. Roy, M. Ortolani, and P. Calvani, Physical Review Letters 121, 176803 (2018).

[12] P. Di Pietro, M. Ortolani, O. Limaj, A. Di Gaspare, V. Giliberti, F. Giorgianni, M. Brahlek, N. Bansal, N. Koirala, S. Oh, et al., Nature Nanotechnology 8, 556 (2013).

[13] C. In, S. Sim, B. Kim, H. Bae, H. Jung, W. Jang, M. Son, J. Moon, M. Salehi, S. Y. Seo, et al., Nano Letters 18, 734 (2018).

[14] J. McIver, D. Hsieh, H. Steinberg, P. Jarillo-Herrero, and N. Gedik, Nature Nanotechnology 7, 96 (2012).

[15] Z. Yue, G. Xue, J. Liu, Y. Wang, and M. Gu, Nature Communications 8, 15354 (2017).

[16] J. A. Sobota, S.-L. Yang, A. F. Kemper, J. Lee, F. T. Schmitt, W. Li, R. G. Moore, J. G. Analytis, I. R. Fisher, P. S. Kirchmann, et al., Physical Review Letters 111, 136802 (2013).

[17] L. Li, W. Xu, and F. Peeters, Journal of Applied Physics 117, 175305 (2015).

[18] N. Bansal, Y. S. Kim, E. Edrey, M. Brahlek, Y. Horibe, K. Iida, M. Tanimura, G.-H. Li, T. Feng, H.-D. Lee, et al., Thin Solid Films 520, 224 (2011).

[19] A. Kuzmenko, Review of Scientific Instruments $\mathbf{7 6}$, 083108 (2005).

[20] See Supplemental Material at [URL will be inserted by the production group] for additional data of the Bi 2 Se 3.

[21] K. Post, B. Chapler, L. He, X. Kou, K. L. Wang, and D. Basov, Physical Review B 88, 075121 (2013).

[22] P. Di Pietro, F. Vitucci, D. Nicoletti, L. Baldassarre, P. Calvani, R. Cava, Y. Hor, U. Schade, and S. Lupi, Physical Review B 86, 045439 (2012).

[23] M. Brahlek, N. Bansal, N. Koirala, S.-Y. Xu, M. Neupane, C. Liu, M. Z. Hasan, and S. Oh, Physical Review Letters 109, 186403 (2012).

[24] M. Salehi, H. Shapourian, N. Koirala, M. J. Brahlek, J. Moon, and S. Oh, Nano Letters 16, 5528 (2016).

[25] L. Wu, M. Brahlek, R. V. Aguilar, A. Stier, C. Morris, Y. Lubashevsky, L. Bilbro, N. Bansal, S. Oh, and N. Armitage, Nature Physics 9, 410 (2013).

[26] S. Sim, N. Koirala, M. Brahlek, J. H. Sung, J. Park, S. Cha, M.-H. Jo, S. Oh, and H. Choi, Physical Review B 91, 235438 (2015).

[27] K. Yu, J. Kim, J. Y. Kim, W. Lee, J. Y. Hwang, E. Hwang, and E. Choi, Physical Review B 94, 235404 (2016).

[28] K. Yu, J. Jeon, J. Kim, C. W. Oh, Y. Yoon, B. J. Kim, J. H. Cho, and E. Choi, Applied Physics Letters 114, 083503 (2019).

[29] K. Yu, B. V. Laun, T. S. Kim, J. Jeon, J. Kim, P. Moon, Y. H. Lee, and E. Choi, Submitted (2019).
[30] N. Bansal, N. Koirala, M. Brahlek, M.-G. Han, Y. Zhu, Y. Cao, J. Waugh, D. S. Dessau, and S. Oh, Applied Physics Letters 104, 241606 (2014).

[31] E. Burstein, Physical Review 93, 632 (1954).

[32] T. Moss, Proceedings of the Physical Society. Section B 67, 775 (1954).

[33] I. Hamberg, C. G. Granqvist, K.-F. Berggren, B. E. Sernelius, and L. Engström, Physical Review B 30, 3240 (1984).

[34] J. G. Analytis, J.-H. Chu, Y. Chen, F. Corredor, R. D. McDonald, Z. Shen, and I. R. Fisher, Physical Review B 81, 205407 (2010).

[35] I. Nechaev, R. Hatch, M. Bianchi, D. Guan, C. Friedrich, I. Aguilera, J. Mi, B. Iversen, S. Blügel, P. Hofmann, and E. Chulkov, Physical Review B 87, 121111 (2013).

[36] Z.-H. Pan, E. Vescovo, A. Fedorov, D. Gardner, Y. Lee, S. Chu, G. Gu, and T. Valla, Physical Review Letters 106, 257004 (2011).

[37] L. A. Wray, S.-Y. Xu, Y. Xia, Y. San Hor, D. Qian, A. V. Fedorov, H. Lin, A. Bansil, R. J. Cava, and M. Z. Hasan, Nature Physics 6, 855 (2010).

[38] A. Dubroka, O. Caha, M. Hronček, P. Friš, M. Orlita, V. Holỳ, H. Steiner, G. Bauer, G. Springholz, and J. Humlíček, Physical Review B 96, 235202 (2017).

[39] B. A. Piot, W. Desrat, D. K. Maude, M. Orlita, M. Potemski, G. Martinez, and Y. S. Hor, Physical Review B 93, 155206 (2016).

[40] I. Aguilera, C. Friedrich, G. Bihlmayer, and S. Blügel, Physical Review B 88, 045206 (2013).

[41] M. Guo, Z. Wang, Y. Xu, H. Huang, Y. Zang, C. Liu, W. Duan, Z. Gan, S.-C. Zhang, K. He, et al., New Journal of Physics 18, 015008 (2016).

[42] T. Förster, P. Krüger, and M. Rohlfing, Physical Review B 91, 035313 (2015).

[43] M. Hermanowicz and M. W. Radny, Physica Status Solidi (RRL)-Rapid Research Letters 13, 1800460 (2019).

[44] W. S. Whitney, V. W. Brar, Y. Ou, Y. Shao, A. R. Davoyan, D. N. Basov, K. He, Q.-K. Xue, and H. A. Atwater, Nano letters 17, 255 (2016). 


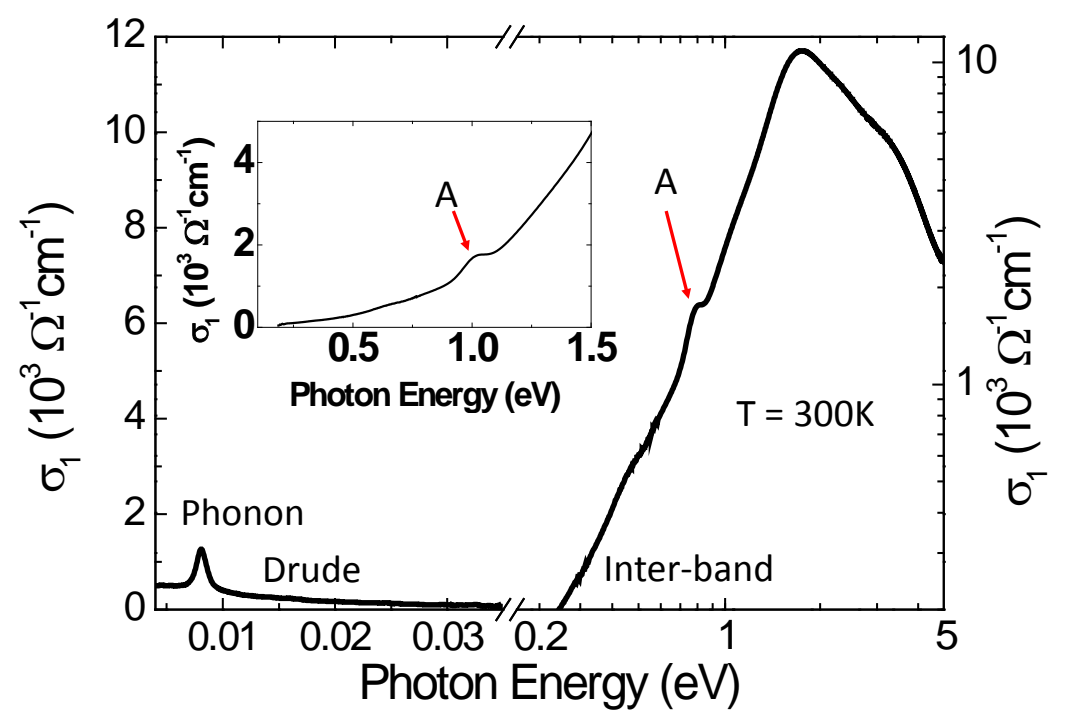

FIG. 1. Optical conductivity of $\mathbf{B i}_{2} \mathbf{S e}_{3}$ thin film (d=50QL) $\sigma_{1}(\omega)$ shows the Drude, phonon, and interband transition. Note that $\log -\log$ scale is used for the $0.2 \mathrm{eV} \leq \hbar \omega \leq 5 \mathrm{eV}$ range. Inset shows the Peak-A in the real scale. 

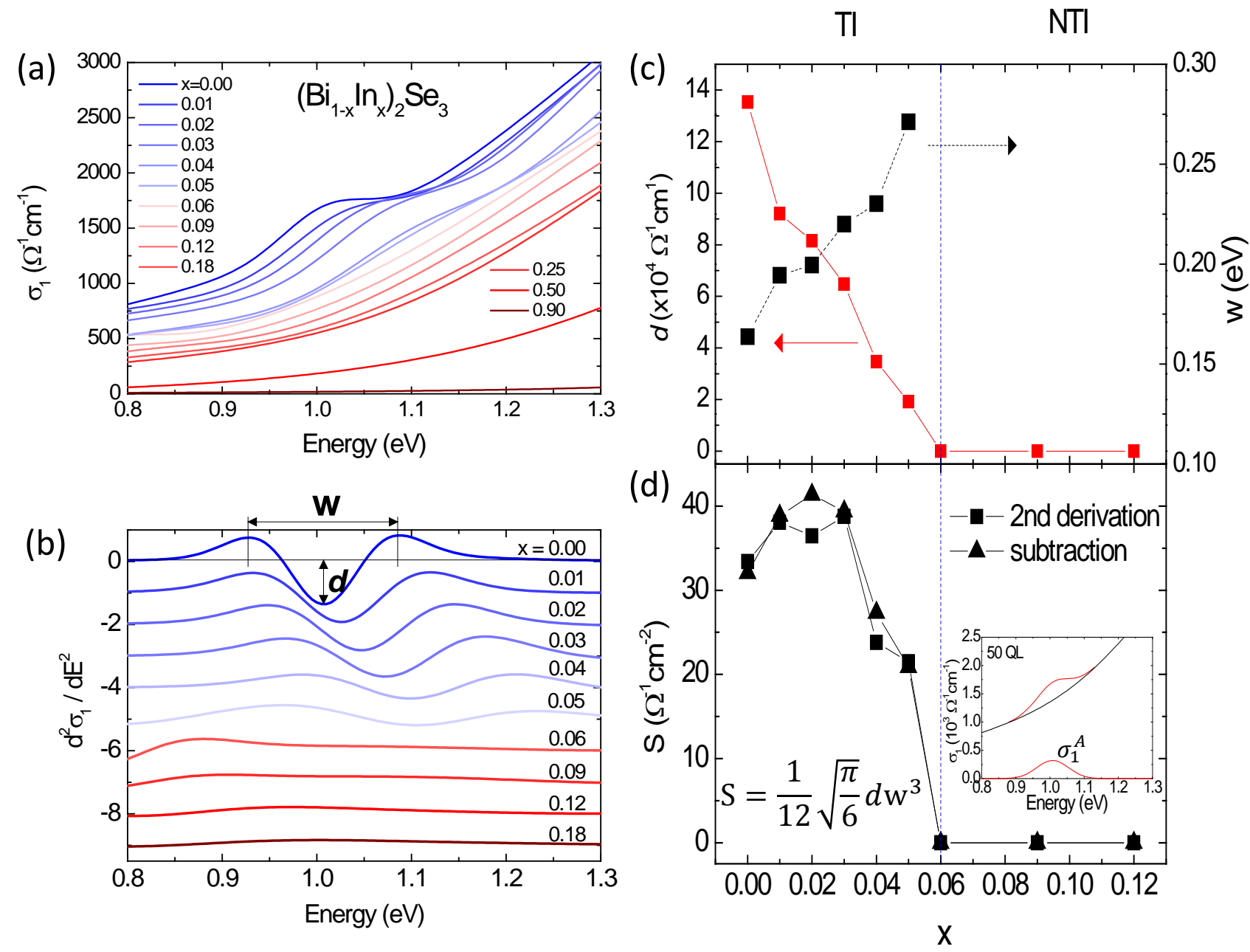

FIG. 2. Evolution of Peak-A in In-substituted $\left(\mathbf{B i}_{1-x} \mathbf{I n}_{x}\right)_{2} \mathbf{S e}_{3}$ thin films. (a) optical conductivity of $\left(\mathrm{Bi}_{1-x} \operatorname{In}_{x}\right)_{2} \mathrm{Se}_{3}$ for the In-concentration range of $0 \leq x \leq 0.9$. (b) The second derivate of optical conductivity $\frac{d^{2} \sigma_{1}}{d \mathrm{E}^{2}}$. The $\mathrm{x}$-dependent behavior of Peak-A can be traced more clearly in this plot. Here $\mathrm{w}$ and $d$ denote the distance between the two maxima and depth of the dip, respectively. (c) The width $\mathrm{w}$ and depth $d$ are shown as function of $\mathrm{x}$. (d) The peak strength $\mathrm{S}$ calculated from $\mathrm{S}=\frac{1}{12} \sqrt{\frac{\pi}{6}} \mathrm{dw}^{3}$ (see Supplemental Fig.S1 20] ). We also calculate $\mathrm{S}$ by $\mathrm{S}=\int \sigma_{1}^{A}(\omega) d \omega$ where $\sigma_{1}^{A}(\omega)=\sigma_{1}(\omega)-\sigma_{1}^{B G}(\omega)\left(\sigma_{1}^{B G}=\right.$ polynomial background) as shown in the inset. In (c) and (d), the critical concentration $\mathrm{x}_{c}=0.06$ of the TI to non-TI (NTI) phase transition is highlighted by the vertical line. 

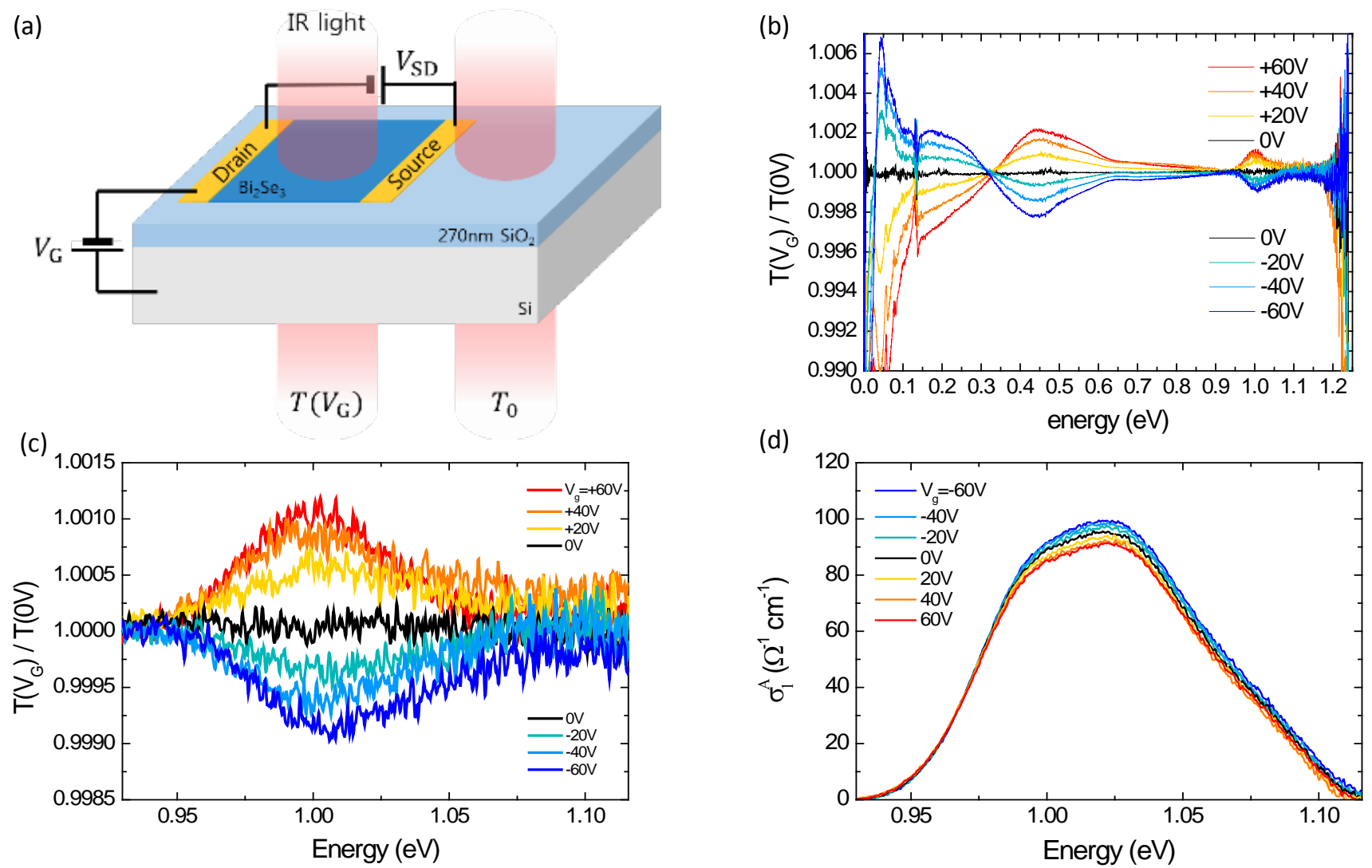

FIG. 3. Gate-driven change of $\mathbf{B i}_{2} \mathbf{S e}_{3}$. (a) Schematic diagram of gate-controlled transmission measurement of $\mathrm{Bi}_{2} \mathrm{Se}_{3}$ thin film. $\mathrm{T}\left(V_{\mathrm{G}}\right)$ is taken with the bias voltage $V_{\mathrm{G}}$ applied between the film and Si. (b) $\mathrm{T}\left(V_{\mathrm{G}}\right) / \mathrm{T}(0)$ changes in the Far-IR, mid-IR, and at $1 \mathrm{eV}$. (c) For peak-A $\mathrm{T}\left(V_{\mathrm{G}}\right)$ increases/decreases for the negative/positive $V_{\mathrm{G}}$, respectively. (d) Optical conductivity of Peak-A, $\sigma_{1}^{A}(\omega)$, was calculated from $\mathrm{T}\left(V_{\mathrm{G}}\right)$ in $(\mathrm{c})$ as described in the text. 

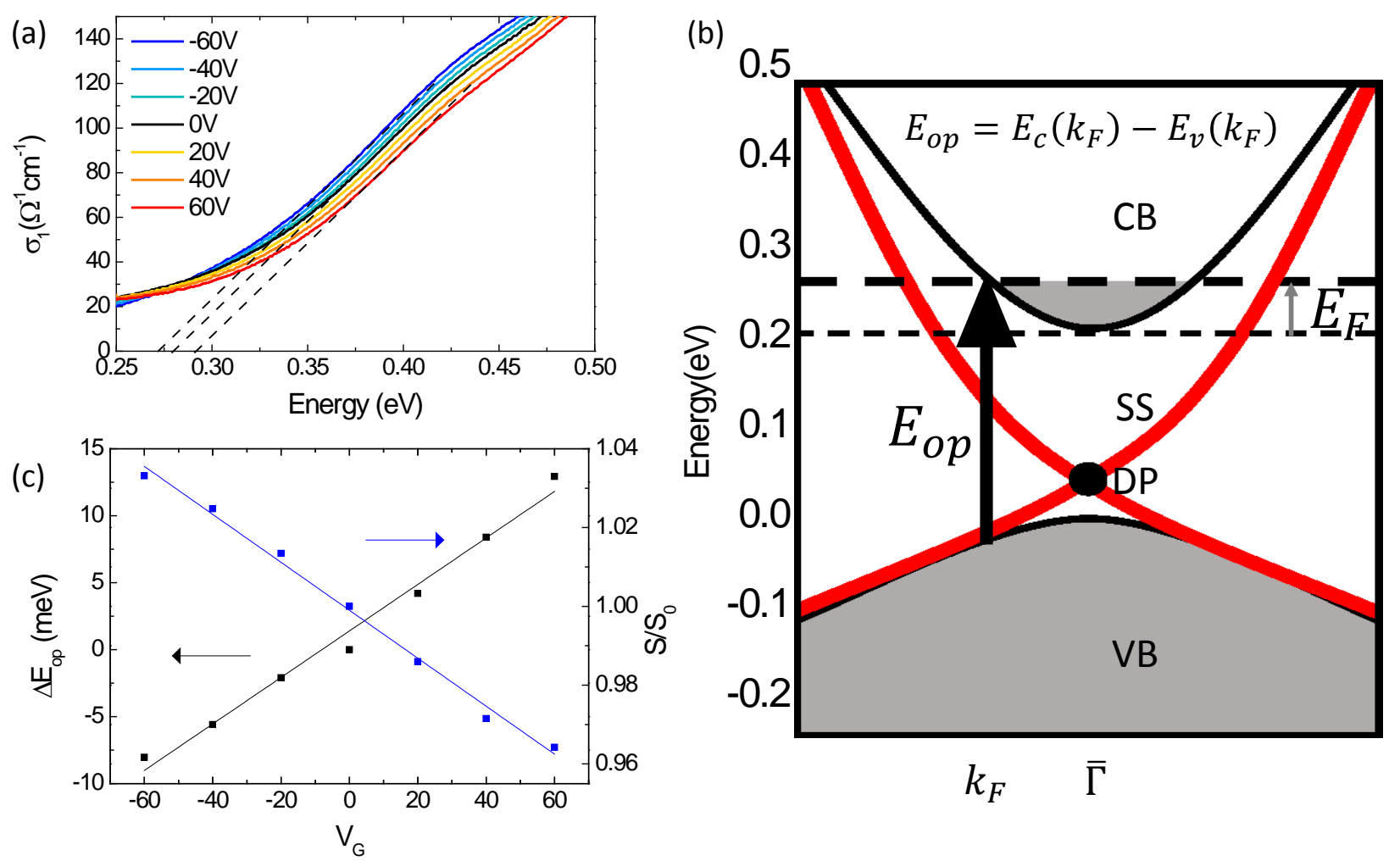

FIG. 4. Estimation of the growth of Peak-A at high gating voltage. (a) Gate-driven shift of the inter-band transition. The onset energy $\mathrm{E}_{o p}$ is determined by the linear extrapolation of data (dashed line). (b) Schematic diagram of the $\mathrm{VB} \rightarrow \mathrm{CB}$ interband transition. The $\mathrm{E}_{o p}$ corresponds to onset of the $\mathrm{VB} \rightarrow \mathrm{CB}$. Here the Fermi energy $\mathrm{E}_{F}$ is measured from the $\mathrm{CB}$ bottom. DP stands for Dirac point. (c) The shift $\Delta \mathrm{E}_{o p}=\mathrm{E}_{o p}\left(V_{\mathrm{G}}\right)-\mathrm{E}_{o p}(0)$ and the change of $S$ are plotted against $V_{\mathrm{G}}$. Here $S$ was calculated by integrating $\sigma_{1}(\omega)$ in Figure 3 c as $S=\int \sigma_{1}^{A}(\omega) d \omega$. 


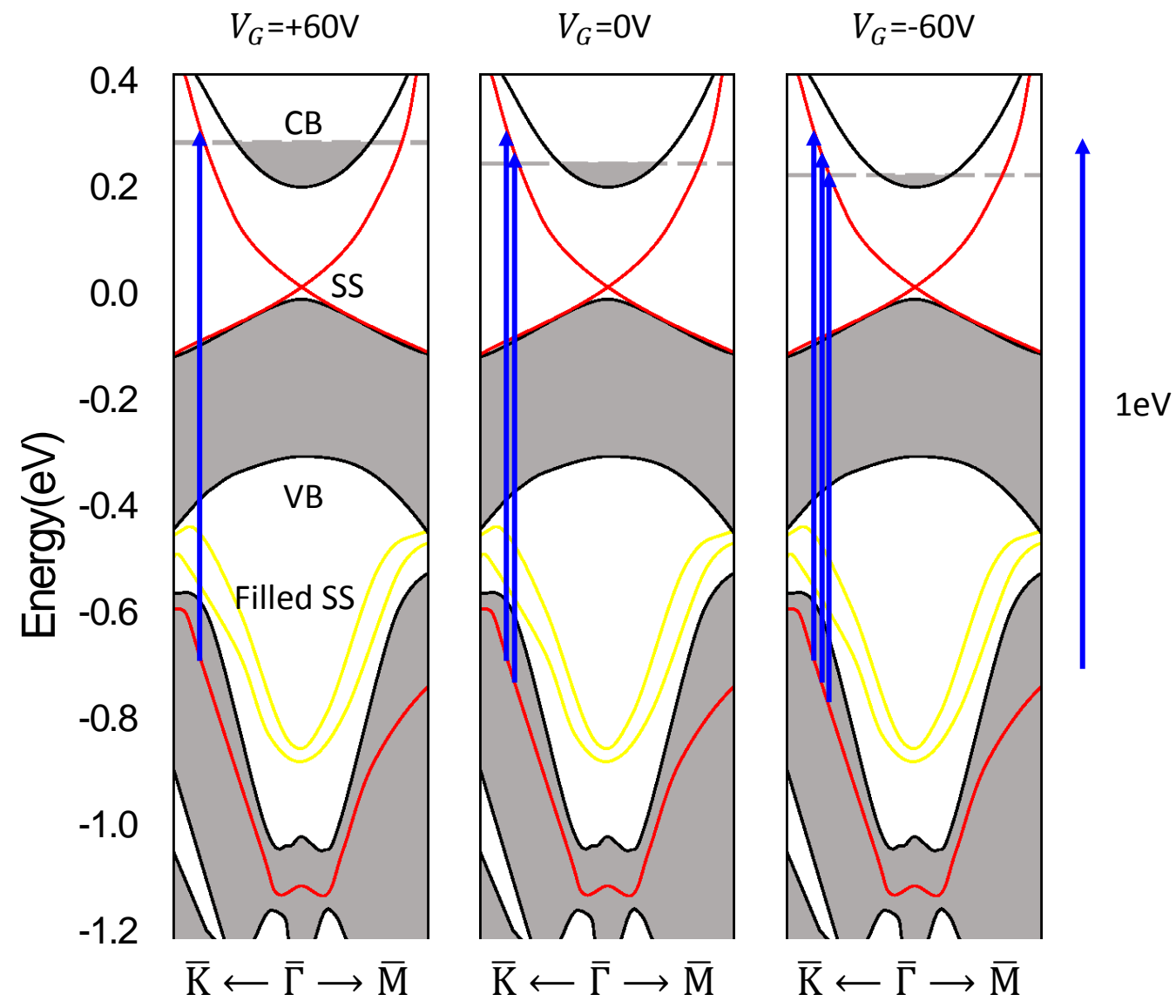

FIG. 5. Schematic band structure and possible origin of Peak-A. For $V_{\mathrm{G}}=0$, electron is excited from the bulk band branch into the empty surface state as highlighted by the blue arrows. For the positive gating, the Fermi level $\mathrm{E}_{F}$ shifts up and the $1 \mathrm{eV}$ - transition becomes weaker. For the negative gating $\mathrm{E}_{F}$ shifts down and the $1 \mathrm{eV}$ - transition becomes stronger. The amount of $\mathrm{E}_{F}$-shift is exaggerated for clarity. The band diagram shown here was redrawn schematically based on experiment [3, 7, 35, 39] and theory 40,43. $\mathrm{CB}=$ bulk conduction band, $\mathrm{VB}=$ bulk valence band, $\mathrm{SS}=$ surface state. 